\title{
A fast discrete small-world optimization algorithm
}

\author{
ZhipengTian $^{1, \text { a }}$, Haiping Zhu ${ }^{1, \mathrm{~b}}$,Xinyu Shao ${ }^{1, \mathrm{c}}$ \\ ${ }^{1}$ Huazhong University of Science \& Technology, Wuhan 430074, China

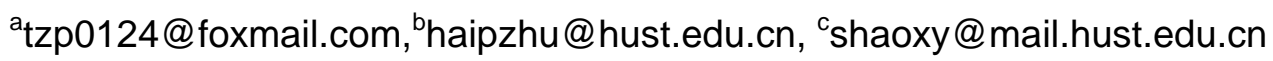

Keywords: Small-world network, discrete small-world optimization, permutation flowshop scheduling problem.

\begin{abstract}
The application of complex networks in optimization field has received increasing attention recently. This paper proposes a discrete small-world optimization algorithm (DSWOA) based on the small-world network theory and Six Degrees of Separation principle in sociology. The DSWOA model is composed of a lot of short-range contacts and few long-range contacts. In this algorithm, the short-range contactsachieve the fast searching and the long-range contacts can accelerate the convergence rate. Next, extensive computational experiments are conducted to compare the DSWOA with other algorithms for the permutation flowshop scheduling problems (PFSP) by using the Taillard instances[1]. In the makespan tests, the results show that DSWOA has a stronger ability of quick searching, especially in the small-size problems. From the analysis results, the DSWOA has higher search efficiency than other algorithms.
\end{abstract}

\section{Introduction and Literature review}

In recent years, the complex network topology, especially the small-world network which is between the regular network and the random network, has gained the interests of many researchers. The earliest research on the small-world originated in 1929 from a Hungarian writer F. Karinthy, who conjectured that in this world any two persons could be related by a chain composed of five contacts[2]. In 1967, S.Milgram confirmed this conjecture by the famous letters delivery experiments and proposed the theory of Six Degrees of Separation [3].

Everyone looks for his target through acquaintances step by step exists in social and economic network widely, which was known as small-world effect[4]. To explain the small-world effect of Six Degrees of Separation, Watts and Strogatz presented the famous concept and model of W-S small-world network[4]. Kleinberg made some improvements to the W-S model. He constructed a new model on the basis of the two-dimensional grid which is more reasonable for the description of small-world phenomenon[5].Then, in order to make the small-world network close to the real social network completely, Watts and Dodds et al. improved the basic W-S model with the multiple categories method[6].

As the small-world network theory system is gradually maturing, it has begun to be used in the optimization field. Walsh studied the application of the small-world network on the problem of graph theory search. He improved the small-world networks to adapt the graph theory search[7]. Chen and Liu et al.pointed out that different values of the spread probability parameters will affect the quick searching feature of small-world network[8]. A small-world topology structure was applied in PSO byCui and Chu et al. to observe the behavior of swarm. This method has made PSO more efficient than before[9]. Li and Zhang et al. proposed a decimal-coding small world optimization algorithm based on Kleinberg's model and demonstrated its stability and fast convertible rate for high-dimensional optimization problems[10]. Li and Shao et al.developed a small-world hierarchical tree model aiming to solve the continuous optimization problem[11].

From these review, there are yet no reports about the application of the small-world network in discrete sequencing optimization problems.Inspired by the application of the small-world effect, this paper proposes a discrete small-world optimization algorithm (DSWOA). In DSWOA, all individuals are connected by short-range and long-range contacts. The short-range contacts help achieve fast search, while the long-range contact can accelerate the convergence rate. In order to verify the DSWOA, the experimental calculation for the permutation flowshop scheduling 
problemis described in Section 3 and the results show that the DSWOA has better searching ability than many other algorithms.

\section{The permutation flowshop scheduling problem}

This paper mainly concerns the discrete optimization sequencing problem called permutation flowshop scheduling problem (PFSP). Flowshops are generally used to model serial manufacturing processes. The flowshop consists of several machines on which jobs are processed in a sequential manner. In the PFSP, all the jobs follow the same processing order on each machines and every job is independent to each other[12].

Suppose there are $n$ jobs and $m$ machines. Let $t(i, j), 1 \leq i \leq n, 1 \leq j \leq m$ be the processing time of job $i$ on the machine $j$ and $\pi=\left(\pi_{1}, \ldots, \pi_{n}\right)$ be a job permutation (i.e., processing order of the jobs). Then the completion times $C\left(\pi_{i}, j\right)$ are calculated as follows:

$$
\begin{aligned}
& C\left(\pi_{0}, j\right)=0, C\left(\pi_{i}, 0\right)=0 \\
& C\left(\pi_{i}, j\right)=\max \left\{C\left(\pi_{i-1}, j\right), C\left(\pi_{i}, j-1\right)+t\left(\pi_{i}, j\right)\right\} ; i=1,2, \ldots, n ; j=1,2, \ldots, m
\end{aligned}
$$

The makespan is $C_{\max }(\pi)=C\left(\pi_{n}, m\right)$.

The objective is to find a permutation $\pi^{*}$ that minimizes $C_{\max }(\pi)$.

\section{Discrete small-world optimization algorithm}

An algorithm with the strong capability of global searching and fast convergence speed is critical to solve the discrete sequencing optimization problems. In this paper, inspired by the intelligent experiments of Milgram's letters delivery[3], we propose a fast discrete small-world optimization algorithm. Under this network structure, while providing some special delivering policies, the optimization of discrete problem will be searched in the small-world network.

In the DSWOA, each solution under consideration is called an envelope node. The basic idea of this algorithm shown in Fig. 1is described as follows:

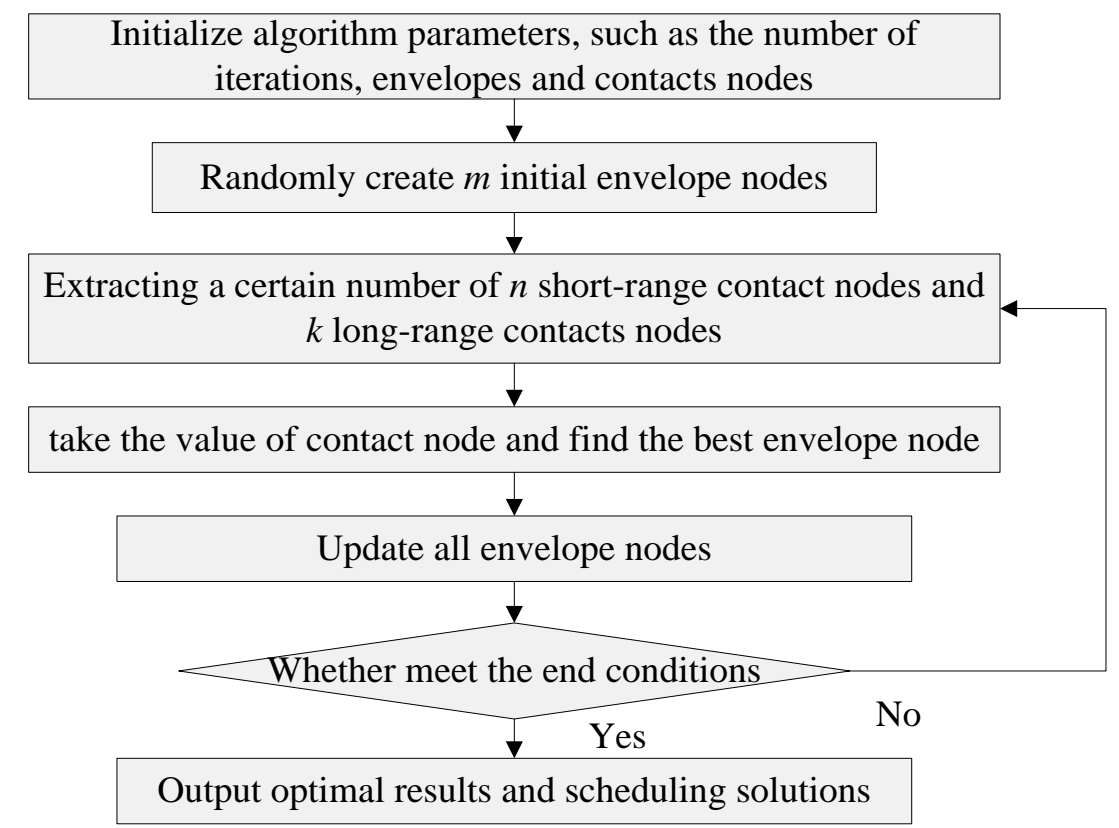

Fig.1 Flowchart of discrete small-world optimization algorithm

Step 1. Set the parameters, including the number of short-range and long-range contact nodes, the iteration times and so on.

Step 2. Initialize the population by randomly generating some solutions (or envelope nodes).

Step 3. Inquire the neighbor nodes. A certain number of short-range and long-range contact 
nodes are inquired for each envelope node in the original solution space and the mapping space.

Step 4. Evaluate the inquired contact nodes by calculating their object values.

Step 5. Deliver envelope nodes. Select the best contact node to replace the current envelope node when it is better than the current envelope node.

Step 6. If the termination criterion is reached, output the best solution; otherwise go to step 3 .

\subsection{Encoding and initialization}

In the DSWOA, supposing $n$ is the number of envelope nodes and $m$ is the coding length of the sequencing problem, for each node, the solution is represented as $X_{i}=\left\{x_{i 1}, X_{i 2}, \ldots, X_{i m}\right\}$ where $x_{i j} \in$ Integer $, i=1,2, \ldots, n, j=1,2, \ldots, m$.

The initial cluster $X_{i}$ is composed of the original envelope nodes, and each node represents candidate solution to the actual problem. The envelopes are delivered following some strategies and will be gradually close to the optimal goal.

\subsection{Definition and selection of short-range and long-range contact}

The small-world network models proposed by Watts and Kleinberg defined the short-rangeand long-range contacts for each individual. In the DSWOA, these two contacts are redefined to search for the optimization objects.

Generally, the Hamming distance is used to measure the relationship for the discrete sequence [13]. For any two different discrete sequences $U=\left\{u_{1}, u_{2}, \ldots, u_{n}\right\}$ and $V=\left\{v_{1}, v_{2}, \ldots, v_{n}\right\}$, their Hamming distance can be computed as $H d=\sum_{i=1}^{n}\left(u_{i} \neq v_{i}\right)$. It is obvious that $2 \leq H d \leq n$. In the DSWOA, we define that two sequences are short-range contact when $H d=2$; the others are long-range contact.

For the discrete sequence, the short-range contact nodes can be obtained with arbitrarily swapping the value in two different positions. Each short-range contact node has an equal opportunity to be selected which is shown in Fig. 2. The large number of short-range contacts can help the DSWOA achieve fast search.

This paper use three different moving methods described in Fig. 3. Operator $M_{2}$ selects a point and inserts it between two adjacent points. Operator $M_{3}$ elects a subsequence of points to insert. Operator $M_{4}$ selects a subsequence of points and relocates these points in the reverse order. For example, when the Hamming distance is selected as $H d=6$ by roulette wheel method, the long-range contact nodes can be selected by any operator method.

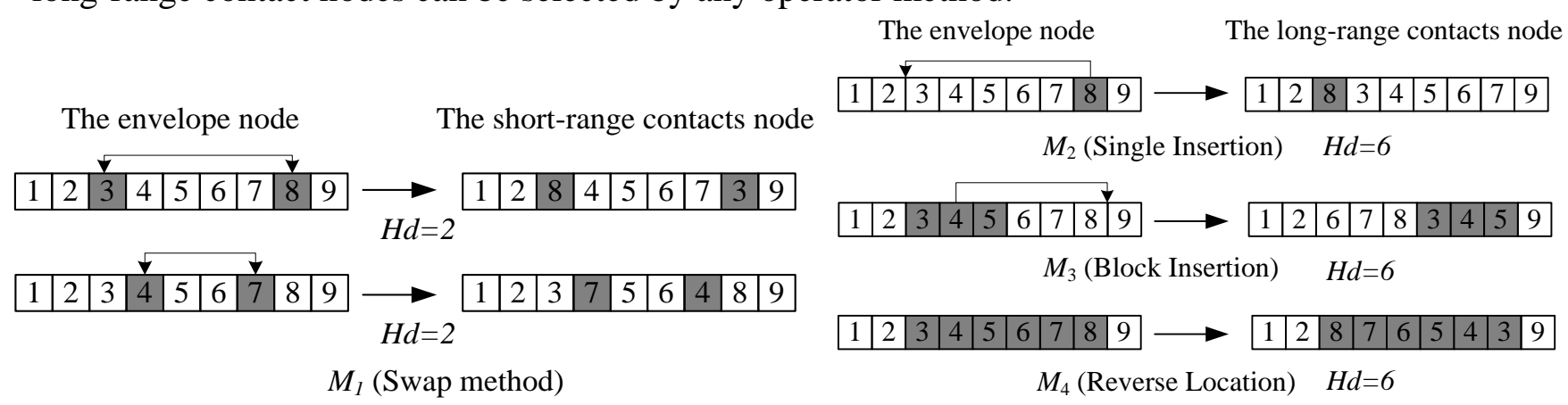

Fig.2The short-range contact nodes

\subsection{Delivering the envelope node}

Fig. 3 Three different long-range contact nodes

In the iterative process of DSWOA, the envelope nodes update in each generation. When delivering the envelope, we select a fixed number of short-range contact nodes generated by the operator $M_{1}$, as well as some long-range contact nodes by the operator $M_{2}, M_{3}$ and $M_{4}$ in Section 3.2. Finally, we compare the selected contact nodes with the envelope node. The envelope node with better objective value replaces the current node. 


\subsection{Experimental results for DSWOA}

To evaluate the effectiveness of DSWOA and compare its performance with other algorithms, in this section we conduct extensive computational experiments for the permutation flowshop scheduling problems (PFSPs) by using the Taillard instances (Taillard, 1993). During the test, the algorithm is realized in Java and the experiments are performed on a PC (Intel Core $2.53 \mathrm{GHz}$ ).

In our experiments, we compare our algorithm with eight other algorithms including Self-guided GA and SGA, ACGA and MGGA, GMA, PSO ${ }_{\mathrm{spv}}$, CPSO and DDE[14, 15]. The parameters of DSWOAare set as: envelope number is $N=50$, the short-range contact nodesnumber is $S N O=30$, the long-range contact nodesnumber is $L N O=10$, and the iteration number is $G=100$. The total of 110 instances are calculated for 10 times independently.

The average error ratio $(E R)$ is used to evaluate the algorithm performance. $E R$ is computed as follows:

$$
E R=\frac{C_{\max }(X)-U}{U},
$$

Where $X$ is the value of makespan generated by an algorithm, whereas $U$ is the value of best known or optimal solution provided by Taillard [1].

We obtain the experimental results based on these tests. Table 1 shows the statistics of the average ER values of all the algorithms on all the 110 test instances. In this table, the bold value is the best solution of each instance in all algorithms. The average CPU time for each instances is 12.62s. The results show that the DSWOA outperforms SGA, MGGA, ACGA, Self-guided GA, $\mathrm{PSO}_{\mathrm{spv}}$, CPSO and GMA in terms of the ER value in most cases. And it is also better than DDE for the small-size problems but inferior to DDE in large-size problems.

From the test results, it's easy to find that the DSWOA has stronger searching ability for the small-size sequence problem. This is primarily due to that individuals have more percentages of short-range contacts in small-size sequencing problems than in large-size sequencing problems. In order to improve the DSWOA's performance in solving large-size sequencing problem, we should consider more domain knowledge such as using local search method or increasing the number of contact nodes to enhance the search ability of DSWOA.

Table 1Average error ratios of all the 9 algorithms on Taillard's instances

\begin{tabular}{ccccccccccc}
\hline $\mathrm{N}$ & $\mathrm{m}$ & SGA & MGGA & ACGA & Self-guided GA & DSWOA & PSO $_{\text {spv }}$ & DDE & CPSO & GMA \\
\hline 20 & 5 & 1.02 & 0.81 & 1.08 & 1.10 & $\mathbf{0 . 0 2}$ & 1.75 & 0.46 & 1.05 & 1.14 \\
& 10 & 1.73 & 1.40 & 1.62 & 1.90 & $\mathbf{0 . 3 7}$ & 3.25 & 0.93 & 2.42 & 2.30 \\
& 20 & 1.48 & 1.06 & 1.34 & 1.60 & $\mathbf{0 . 3 7}$ & 2.82 & 0.79 & 1.99 & 2.01 \\
50 & 5 & 0.61 & 0.44 & 0.57 & 0.52 & $\mathbf{0 . 1 7}$ & 1.14 & $\mathbf{0 . 1 7}$ & 0.90 & 0.47 \\
& 10 & 2.81 & 2.56 & 2.79 & 2.74 & $\mathbf{1 . 9 7}$ & 5.29 & 2.26 & 4.85 & 3.21 \\
& 20 & 3.98 & 3.82 & 3.75 & 3.94 & 3.27 & 7.21 & $\mathbf{3 . 1 1}$ & 6.40 & 4.97 \\
100 & 5 & 0.47 & 0.41 & 0.44 & 0.38 & 0.23 & 0.63 & $\mathbf{0 . 0 8}$ & 0.74 & 0.42 \\
& 10 & 1.67 & 1.50 & 1.71 & 1.60 & 1.18 & 3.27 & $\mathbf{0 . 9 4}$ & 2.94 & 1.96 \\
& 20 & 3.80 & $\mathbf{3 . 1 5}$ & 3.47 & 3.51 & 3.90 & 8.25 & 3.24 & 7.11 & 4.68 \\
200 & 10 & 0.94 & 0.92 & 0.94 & $\mathbf{0 . 8 0}$ & 0.96 & 2.47 & $\mathbf{0 . 5 5}$ & 2.17 & 1.10 \\
& 20 & 2.73 & 3.95 & 2.61 & $\mathbf{2 . 3 2}$ & 3.95 & 8.05 & 2.61 & 6.89 & 3.61 \\
& Total & 1.93 & 1.82 & 1.85 & 1.85 & 1.49 & 4.01 & $\mathbf{1 . 3 8}$ & 3.40 & 2.35 \\
\hline
\end{tabular}

\section{Conclusions}

Inspired by the small-world network theory and the Six Degrees of Separation,we propose a discrete small-world optimization algorithm and use it to solve the discrete sequencing problem. This algorithm is suitable for heuristic methods to search targets efficiently. The large of short-range contacts help the DSWO fast searching and the long-range contacts can accelerate the convergence rate. The experimental results of minimizing the makespan show that the DSWOA is very promising. It outperforms SGA, MGGA, ACGA, Self-guided GA, PSOvns, CPSO and GMA in terms of the $E R$ value. These test results sufficiently show that DSWOA has a strong ability of quick searching, especially in the small-size sequence problem. 
In the future, we will continue to improve the DSWOA for discrete problem and combine more local search methods to perfect the solving performance in large-size problem. And we also plan to extend the applicability of the discrete small-world optimization algorithm to other scheduling problems, sequentially make the DSWOA be applied in more extensive fields.

\section{Acknowledgements}

The authors greatly acknowledge the financial supports from the National Science and Technology Major Project of China under the Grant No. 2014ZX04002011 and 2014ZX04014101.

\section{References}

[1]. Taillard, E., Benchmarks for basic scheduling problems. european journal of operational research, 1993. 64(2): p. 278-285.

[2]. Braun, T., Hungarian priority in network theory. Science (New York, NY), 2004. 304(5678): p. 1745.

[3]. Milgram, S., The small world problem. Psychology today, 1967. 2(1): p. 60-67.

[4]. Watts, D.J. and S.H. Strogatz, Collective dynamics of small-world networks. nature, 1998. 393(6684): p. 440-442.

[5]. Kleinberg, J.M., Navigation in a small world. Nature, 2000. 406(6798): p. 845-845.

[6]. Watts, D.J., P.S. Dodds and M.E. Newman, Identity and search in social networks. science, 2002. 296(5571): p. 1302-1305.

[7]. Walsh, T. Search in a small world. in IJCAI. 1999.

[8]. Chen, J., W. Liu and J. Zhu, Two-dimensional small-world networks: Navigation with local information. Physical Review E, 2006. 73(5): p. 056111.

[9]. Cui, Z., Y. Chu and X. Cai, Nearest neighbor interaction PSO based on small-world model, in Intelligent Data Engineering and Automated Learning-IDEAL 2009. 2009, Springer. p. 633-640.

[10].Li, X., et al. A small world algorithm for high-dimensional function optimization. in Computational Intelligence in Robotics and Automation (CIRA), 2009 IEEE International Symposium on. 2009.

[11].Li, J., et al., Global optimization by small-world optimization algorithm based on social relationship network. Journal of Central South University, 2012. 19(8): p. 2247-2265.

[12].Tasgetiren, M.F., et al., A particle swarm optimization algorithm for makespan and total flowtime minimization in the permutation flowshop sequencing problem. European Journal of Operational Research, 2007. 177(3): p. 1930-1947.

[13].Norton, G.H. and A. Salagean, On the Hamming distance of linear codes over a finite chain ring. Information Theory, IEEE Transactions on, 2000. 46(3): p. 1060-1067.

[14].Pan, Q., M.F. Tasgetiren and Y. Liang, A discrete differential evolution algorithm for the permutation flowshop scheduling problem. Computers \& Industrial Engineering, 2008. 55(4): p. 795-816.

[15].Chen, S., et al., A self-guided genetic algorithm for permutation flowshop scheduling problems. Computers \& Operations Research, 2012. 39(7): p. 1450-1457. 